\title{
MicroRNA-195-5p is associated with cell proliferation, migration and invasion in prostate cancer and targets MIB1
}

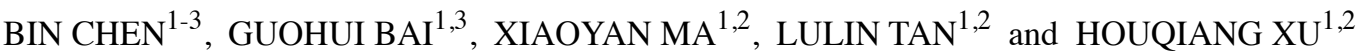 \\ ${ }^{1}$ College of Life Sciences; ${ }^{2}$ Key Laboratory of Animal Genetics, Breeding and Production in \\ The Plateau Mountains Region, Ministry of Education, Guizhou University, Guiyang, \\ Guizhou 550025; ${ }^{3}$ Zunyi Medical University, Zunyi, Guizhou 563000, P.R. China
}

Received April 30, 2021; Accepted August 18, 2021

DOI: $10.3892 / o r .2021 .8210$

\begin{abstract}
Mind bomb 1 (MIB1) is a well-known E3 ubiquitin ligase. MicroRNAs (miRNAs/miRs) have been found to serve important functions in cancer cell physiology. However, the clinical significance and biological function of MIB1 and miRNAs in prostate cancer (PCa) are yet to be fully elucidated. The current study predicted the interaction between MIB1 and miR-195-5p using TargetScan, and the results were confirmed by performing a dual-luciferase reporter assay. The mRNA expression level of MIB1 and miR-195-5p in PCa and adjacent normal tissues, and PCa cell lines was detected using reverse transcription-quantitative PCR. Cell Counting Kit- 8 and Transwell assays were used to measure the proliferation, and migration and invasion of $\mathrm{VCaP}$ and DU145 PCa cell lines, respectively, while western blot analysis was used to detect the protein expression level of MIB1. The results revealed that the mRNA expression level of MIB1 was increased, while the mRNA expression level of miR-195-5p was decreased in PCa tissues ( $\mathrm{P}<0.001$ and $\mathrm{P}<0.01$, respectively) and in various cell lines, including $\mathrm{PC}-3(\mathrm{P}<0.001$ and $\mathrm{P}<0.05$, respectively), $\mathrm{VCaP}$ $(\mathrm{P}<0.001$ and $\mathrm{P}<0.01$, respectively), 22Rv1 $(\mathrm{P}<0.001$ and $\mathrm{P}<0.05$, respectively), DU145 ( $\mathrm{P}<0.001$ and $\mathrm{P}<0.01$, respectively) and $\mathrm{LNCaP}(\mathrm{P}<0.001$ and $\mathrm{P}<0.05$, respectively). miR-195-5p mimics rescued the inhibitory effects caused by knockdown of MIB1 on cell proliferation, migration and invasion in the $\mathrm{VCaP}$ and DU145 cell lines. In addition, MIB1 overexpression restored the miR-195-5p overexpression-induced repression of cell proliferation and invasion. The current study revealed that the MIB1 gene was an effector of cell proliferation, migration and invasion in PCa cell lines. Furthermore, miR-195-5p may regulate PCa cell proliferation and invasion by regulating MIB1, indicating its potential therapeutic application for $\mathrm{PCa}$ in the future.
\end{abstract}

Correspondence to: Professor Houqiang $\mathrm{Xu}$, College of Life Sciences, Guizhou University, 2708 South Section of Huaxi Avenue, Guiyang, Guizhou 550025, P.R. China

E-mail: xuhouqiangyx@163.com

Key words: prostate cancer, microRNA-195-5p, mind bomb 1, proliferation, migration, invasion

\section{Introduction}

Prostatecancer ( $\mathrm{PCa}$ ) has becomea leading cause of cancer-related mortality among men worldwide (1-3). In addition, as the population ages, prostate cancer rates are likely to increase (4). The progression of $\mathrm{PCa}$ to hormone-dependent disease is associated with advanced metastasis (5). As such, the 5-year survival rate of most patients with a PCa diagnosis is $>90 \%$; however, the survival rate of patients with metastatic disease is only $29 \%$ (6). A number of patients are diagnosed with $\mathrm{PCa}$ at an advanced stage where treatment is usually less effective (7). In addition, due to the high incidence rate of overdiagnosis and overtreatment using prostate-specific antigen $(8,9)$, there is an urgent requirement to improve the understanding of the molecular mechanisms underlying PCa to assist in the development of new diagnostic and prognostic markers, and treatment strategies.

Mind bomb 1 (MIB1) is a well-known E3 ubiquitin ligase (10). Previous studies have primarily focused on its ubiquitination in various pathways. For example, in 2017, Mizoguchi et al (10) demonstrated that MIB1 ubiquitinated CTNND1, with MIB1 contributing to persistent directional cell migration by regulating the CTNND1/Rac1 pathway. In addition, Berndt et al (11) reported that MIB1 served a role in the regulation of the Wnt/ $\beta$-catenin signaling pathway via the ubiquitination and degradation of receptor-like tyrosine kinase. MIB1 has been reported to regulate apoptosis by interacting with death-associated protein kinase (12) and cellular FLICE-like inhibitory protein (13). Furthermore, it was revealed that MIB1 was necessary for the efficient activation of Notch signaling via the ubiquitination of Notch receptors (14). However, little is known on the interaction between MIB1 and microRNAs (miRNAs/miRs) in cancer, with only Ray et al (15) demonstrating that MIB1 knockdown recapitulated the effects of miR-198 on the proliferation and colony formation of DU145 and LNCaP cells lines.

miRNAs are small non-coding RNAs, 18-22 nucleotides in length, that serve important functions in cell physiology by regulating the expression of mRNAs (16). An increasing amount of evidence has suggested that miR-195-5p functions as a tumor suppressor in numerous types of cancer, including non-small cell lung (NSCLC), cervical and prostate cancers (17-24). For example, in NSCLC, miR-195-5p overexpression inhibited cell proliferation and induced apoptosis by directly targeting CIAPIN1 (17) and CEP55 (18), and inhibited cell proliferation, migration, and 
invasion by directly targeting CPNE1 (19). Liu et al (20) demonstrated that miR-195-5p inhibited the tumor development of cervical cancer by targeting YAP1. The current study investigated the expression pattern, role and potential functional mechanism of MIB1 and miR-195-5p in PCa. The association between MIB1 and miR-195-5p was then further determined in the context of PCa cell proliferation, migration and invasion.

\section{Materials and methods}

Clinical sample selection and ethics statement. Surgically resected $\mathrm{PC}$ a tissues and adjacent normal tissues (distance, $>3 \mathrm{~cm}$ ) were collected from 40 patients with PCa between March 2018 and November 2019 at the Affiliated Hospital of Zunyi Medical University (Guizhou, China). All 40 patients were pathologically confirmed. Pathological grading was based on Gleason scoring in accordance with the World Health Organization histopathological classification standard for prostate cancer $(25,26)$. Exclusion criteria were as follows: i) A history of preoperative radiotherapy or chemotherapy; ii) benign prostatic hyperplasia or urolithiasis and iii) a history of other types of cancer or systemic immune disease. The clinical data of these patients were collected from their medical records and are presented in Table SI. None of the patients received radiotherapy or chemotherapy prior to resection. The current research was approved by the Ethics Committee of Zunyi Medical University (Guizhou, China) and all patients provided written informed consent. All PCa tissues and adjacent normal tissues were immediately snap-frozen in liquid nitrogen and stored at $-80^{\circ} \mathrm{C}$.

Cell culture and transfection. The PCa cell lines, including PC-3 [American Type Culture Collection (ATCC) no. CRL-1435)], VCaP (ATCC no. CRL-2876), 22Rv1 (ATCC no. CRL-2505), DU145 (ATCC no. HTB-81) and LNCaP (ATCC no. CRL-1740), along with the normal human prostate epithelial cell line RWPE1 (ATCC no. CRL-11609), were purchased from the ATCC. The RWPE1 cell line was cultured in Keratinocyte Serum-Free medium (Gibco; Thermo Fisher Scientific, Inc.), while the PC-3, VCaP, 22Rv1, DU145 and LNCaP cell lines were maintained in minimum essential medium (MEM) supplemented with 10\% FBS (Gibco; Thermo Fisher Scientific, Inc.) at $37^{\circ} \mathrm{C}$ in a humidified incubator with $5 \% \mathrm{CO}_{2}$.

The primer sequences for cell transfection were synthesized by Shanghai GenePharma Co., Ltd. The cells were divided into groups according to their treatments as follows: $50 \mathrm{nM}$ miR-195-5p mimics (transfection with miR-195-5p mimics; 5'-UAGCAGCACAGAAAUAUUGGC-3' and 5'-CAAUAU UUCUGUGCUGCUAUU-3'), $50 \mathrm{nM}$ miR-negative control (NC; non-targeting; transfection with miR-195-5p mimics NC; 5'-UCACAACCUCCUAGAAAGAGUAGA-3' and 5'-CAG UACUUUUGUGUAGUACAA-3'); 50 nM anti-miR-195-5p (transfection with miR-195-5p inhibitor; 5'-GCCAAUAUU UCUGUGCUGCUA-3'), $50 \mathrm{nM}$ anti-miR-NC (transfection with NC miR-195-5p inhibitor; 5'-CAGUACUUUUGUGUAGUA CAA-3'); $2 \mu \mathrm{g}$ short hairpin (sh) RNA targeting MIB1 (transfection with sh-MIB1 plasmid; 5'-GGAUAAAGAUGGUGAUAG ATT-3' and 5'-UCUAUCACCAUCUUUAUCCTT-3'), $2 \mu \mathrm{g}$ sh-NC (non-targeting; transfection with NC sh-MIB1 plasmid; 5'-UUCUCCGAACGUGUCACGUTT-3' and 5'-ACGUGA CACGUUCGGAGAATT-3'); $2 \mu$ g overexpression (OE)-MIB1 (transfection with OE-MIB1 plasmid), $2 \mu \mathrm{g}$ OE-NC (transfection with OE-MIB1 NC plasmid); miR-195-5p mimic + OE-NC (transfection with miR-195-5p mimic and OE-NC plasmid), miR-195-5p mimic + OE-MIB1 (transfection with miR-195-5p mimic and OE-MIB1 plasmid); anti-miR-195-5p + sh-MIB1 (transfection with miR-195-5p inhibitor and sh-MIB1) and anti-miR-195-5p + sh-NC (transfection with miR-195-5p inhibitor and sh-NC). Full length MIB1 sequences were amplified via PCR and subcloned into pcDNA3.1 vector (Invitrogen; Thermo Fisher Scientific, Inc.) to generate OE MIB1 plasmids. The recombinant plasmid vectors expressing the double-stranded shRNA targeting MIB1 (sh-MIB1) or the shRNA control (sh-NC) were purchased from Shanghai GenePharma Co., Ltd. The PCa cells were seeded into a 6 -well plate $\left(1 \times 10^{6}\right.$ cells/well $)$ and the aforementioned oligonucleotides were transfected into each PCa cell line using Lipofectamine ${ }^{\circledR} 3000$ transfection reagent (Invitrogen; Thermo Fisher Scientific, Inc.) at $37^{\circ} \mathrm{C}$ for $48 \mathrm{~h}$, according to the manufacturer's protocol. At $48 \mathrm{~h}$ post transfection, the transfection efficiency was detected using reverse transcription-quantitative PCR (RT-qPCR).

Total RNA extraction and reverse transcription-quantitative $P C R$ (RT-qPCR). Total RNA was isolated from tumor tissues and cell lines using TRIzol ${ }^{\circledR}$ (Invitrogen, Thermo Fisher Scientific, Inc.) in accordance with the manufacturer's protocol. The concentration and purity of the extracted RNA was then determined at an absorbance ratio of 260/280 nm using a NanoDrop 2000 spectrophotometer (Invitrogen; Thermo Fisher Scientific, Inc.). Subsequently, $1 \mu \mathrm{g}$ RNA was reverse transcribed into cDNA using the PrimeScript ${ }^{\mathrm{TM}}$ $\mathrm{RT}$ reagent kit (Takara Bio, Inc.) according to the manufacturer's protocol. qPCR reactions were performed using a SYBR ${ }^{\circledR}$ Premix Ex Taq ${ }^{\mathrm{TM}}$ II kit (Takara Bio, Inc.) with an ABI 7500 real-time PCR system (Applied Biosystems; Thermo Fisher Scientific, Inc.). The following thermocycling conditions were used: Initial denaturation at $95^{\circ} \mathrm{C}$ for $5 \mathrm{~min}$, followed by 40 cycles at $95^{\circ} \mathrm{C}$ for $1 \mathrm{~min}, 60^{\circ} \mathrm{C}$ for $30 \mathrm{sec}$ and $72^{\circ} \mathrm{C}$ for $30 \mathrm{sec}$. A final cycle was implemented at $72^{\circ} \mathrm{C}$ for 5 min. GAPDH and U6 small nuclear RNA served as internal controls for MIB1 and miR-195-5p expression, respectively. The primers used for qPCR are listed in Table I. Relative expression levels were calculated using the $2^{-\Delta \Delta \mathrm{Cq}}$ method (27).

Cell proliferation, migration and invasion analyses. The proliferation of the PCa cell lines was evaluated using a Cell Counting Kit-8 (CCK-8) assay (Beyotime Institute of Biotechnology). Following transfection, the VCaP and DU145 cell lines were cultured in 96-well plates, at a density of $1 \times 10^{6}$ cells/well, and incubated for $0,24,48$ or $72 \mathrm{~h}$ before adding $10 \mu \mathrm{l} \mathrm{CCK}-8$ solution to each well. The cells were subsequently incubated at $37^{\circ} \mathrm{C}$ for $2 \mathrm{~h}$. Cell proliferation was measured using a microplate reader at an absorbance of $450 \mathrm{~nm}$ (Thermo Fisher Scientific, Inc.).

The migration and invasion of the PCa cell lines was assessed using Transwell and Matrigel assays. Following transfection, the VCaP and DU145 cell lines were seeded into the upper chamber at a density of $1 \times 10^{5}$ cells/well and then incubated for $24 \mathrm{~h}$ at $37^{\circ} \mathrm{C}$. The Transwell chambers were pre-coated with $100 \mu 1$ Matrigel (BD Biosciences) for the evaluation of cell invasion at $37^{\circ} \mathrm{C}$ for $1 \mathrm{~h}$. The lower chamber was filled with $500 \mu \mathrm{l}$ MEM supplemented with $10 \%$ FBS. The 
Table I. Primer sequences for reverse transcription-quantitative PCR.

\begin{tabular}{lll}
\hline Name & \multicolumn{1}{c}{ Forward sequence (5'-3') } & \multicolumn{1}{c}{ Reverse sequence (5'-3') } \\
\hline miR-195-5p & ACACTCCAGCTGGGTAGCAGCACAGAAAT & TGGTGTCGTGGAGTCG \\
MIB1 & TAACCGGGTGATGGTGGAAG & GTGCCGTTGTCCCACACTA \\
GAPDH & TCAAGGCTGAGAACGGGAAG & TCGCCCCACTTGATTTGGA \\
U6 & ATTGGAACGATACAGAGAAGATT & GGAACGCTTCACGAATTTG \\
\hline
\end{tabular}

PCa cells that migrated or invaded through the membranes into the lower chamber were fixed with $100 \%$ methanol for $10 \mathrm{~min}$ at room temperature and stained with $0.5 \%$ crystal violet solution for $10 \mathrm{~min}$ at room temperature. Finally, the number of migrated and invaded cells was determined using a light microscope (Olympus Corporation) in three randomly selected fields of view. The statistical analysis of cell counts was performed using ImageJ 1.47v software (National Institutes of Health).

Dual-luciferase reporter assay. Potential binding sites between MIB1 and miR-195-5p were identified using TargetScan (http:// www.targetscan.org/). MIB1 sequences containing the binding sites [MIB1 wild-type (WT)] and non-binding sites [MIB1 mutant (MUT)] of miR-195-5p were amplified and subcloned into a psiCHECK2 luciferase vector (Promega Corporation). The MIB1-WT or MIB1-MUT reporter vector was subsequently co-transfected into the VCaP and DU145 cell lines using with the aforementioned miR-195-5p mimics and its corresponding NC Lipofectamine ${ }^{\circledR} 3000$ (Invitrogen; Thermo Fisher Scientific, Inc.). Following culture for 48 h, luciferase activity was examined using the Dual-Luciferase Reporter Assay kit (Promega Corporation). Subsequently, the luciferase activity was normalized to the firefly luciferase internal control.

Western blot analysis. The tumor tissues or PCa cell lines were harvested and lysed using RIPA buffer (Beijing Solarbio Science and Technology Co., Ltd.). The protein concentration was quantified using a BCA protein assay kit (Sangon Biotech Co., Ltd.). Then, protein samples (30 $\mu \mathrm{g}$ per lane) were separated using $12 \%$ SDS-PAGE and transferred onto PVDF membranes following electrophoresis for $2 \mathrm{~h}$. The membranes were subsequently blocked using $5 \%$ skimmed milk in TBS and $0.1 \%$ Tween-20 (TBST) at $37^{\circ} \mathrm{C}$ for $2 \mathrm{~h}$, following which they were incubated with GAPDH $(1: 1,000$; cat. no. 3781) or MIB1 (1:1,000; cat. no. 1202) (both from Generay Biotech Co., Ltd.) primary antibodies overnight at $4^{\circ} \mathrm{C}$. After washing with TBST three times, the membranes were incubated with corresponding horseradish peroxidase-conjugated secondary antibodies (1:10,000; cat. no. ZB-2306; Beijing Zhongshan Golden Bridge Biotechnology Co., Ltd.) for $1 \mathrm{~h}$ at room temperature. The signals were visualized using the Pierce ${ }^{\mathrm{TM}}$ ECL Western Blotting Substrate (Thermo Fisher Scientific, Inc.) and quantified using Image J v1.8.0 software (National Institutes of Health).

Statistical analysis. Statistical analysis was performed using SPSS v22.0 (IBM Corp.) and GraphPad Prism v8.0 (GraphPad Software, Inc.). All experiments were performed in triplicate and the data are presented as the mean \pm SD. Differences between tumor tissues and their adjacent normal tissues were compared using a paired Student's t-test. Unpaired Student's t-test was used for comparisons between unpaired groups and one-way ANOVA followed by Tukey's post hoc test was used for comparisons among multiple groups. The association between miR-195-5p and MIB1 expression was analyzed using the Pearson's correlation coefficient. $\mathrm{P}<0.05$ was considered to indicate a statistically significant difference.

\section{Results}

MIB1 expression is upregulated in PCa tissues and cell lines. RT-qPCR was conducted to detect the mRNA expression level of MIB1 in PCa tissues and cell lines. Compared with that in the adjacent normal tissues, the mRNA expression level of MIB1 was significantly increased in PCa tissues $(n=40$; Fig. 1A). Furthermore, the results of RT-qPCR and western blot analysis revealed that MIB1 mRNA and protein expression level was markedly increased in the PCa cell lines (PC-3, VCaP, 22Rv1, DU145 and LNCaP) compared with that in the normal human prostate epithelial cell line, RWPE1, respectively (Fig. 1B and C). The results suggested that MIB1 may serve a role in the occurrence and development of $\mathrm{PCa}$.

MIB1 overexpression markedly promotes PCa cell proliferation, migration and invasion. Due to the higher expression level of MIB1 in the VCaP and DU145 cell lines when compared with that in the other three PCa cell lines, they were selected for further functional analysis. To determine the effect of MIB1 on the proliferation, migration and invasion of PCa cells, the VCaP and DU145 cell lines were transfected with OE-MIB1, sh-MIB1, OE-NC or sh-NC. As presented in Fig. 2A, the mRNA and protein expression level of MIB1 was notably increased in the VCaP and DU145 cell lines following OE-MIB1 transfection. However, the expression level was markedly decreased following transfection with sh-MIB1. The CCK-8 assay results indicated that, compared with that in the sh-NC group, the stable knockdown of MIB1 significantly inhibited the proliferation of the $\mathrm{VCaP}$ and DU145 cell lines, with MIB1 overexpression significantly promoting the proliferation of the VCaP and DU145 cell lines compared with that in the OE-NC group (Fig. 2B). The results of the Transwell and Matrigel assays confirmed that the inhibition of MIB1 markedly repressed the mobility and invasiveness of the VCaP and DU145 cell lines, and that MIB1 overexpression significantly promoted cell migration and invasion (Fig. 2C and D). These data suggested that MIB1 may promote the proliferation, migration and invasion of PCa cells.

MIB1 is targeted by miR-195-5p. To further investigate the regulatory network of MIB1 in PCa, TargetScan was used 

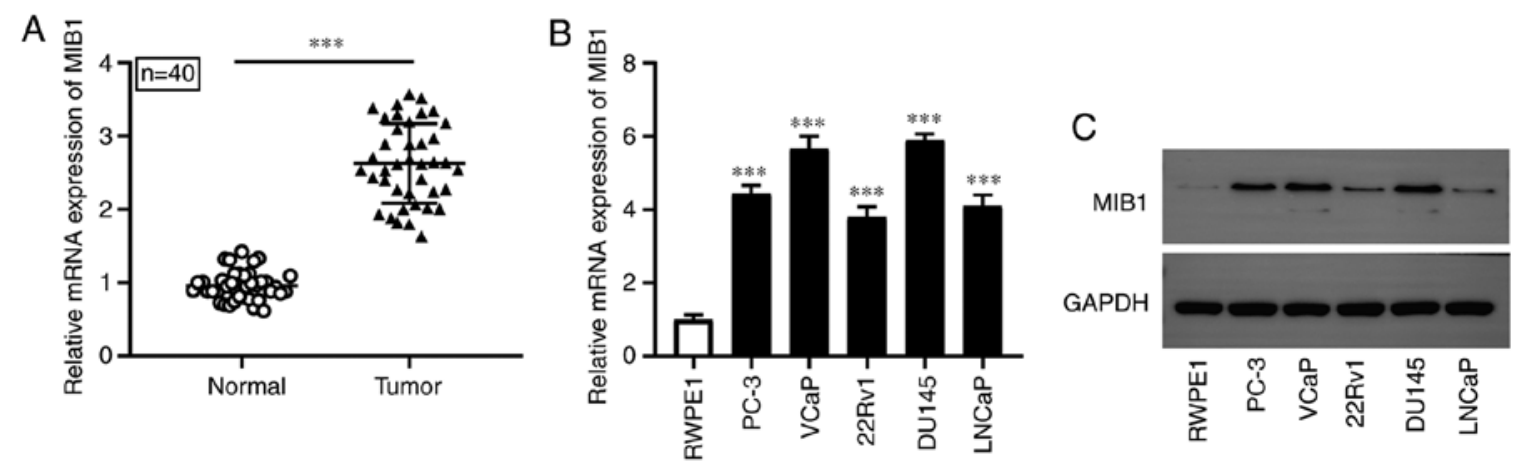

Figure 1. MIB1 is highly expressed in PCa tissues and cell lines. The mRNA expression level of MIB1 was measured in (A) PCa tissues and adjacent normal tissues ( $\mathrm{n}=40)$, and in (B) PCa cell lines (PC-3, VCaP, 22Rv1, DU145 and LNCaP) and a normal human prostate epithelial cell line (RWPE1) using reverse transcription-quantitative PCR analysis. ${ }^{* * *} \mathrm{P}<0.001$. (C) Western blot analysis of MIB1 protein expression level in PCa cell lines (PC-3, VCaP, 22Rv1, DU145 and $\mathrm{LNCaP}$ ) and a normal human prostate epithelial cell line (RWPE1). MIB1, mind bomb 1; PCa, prostate cancer.
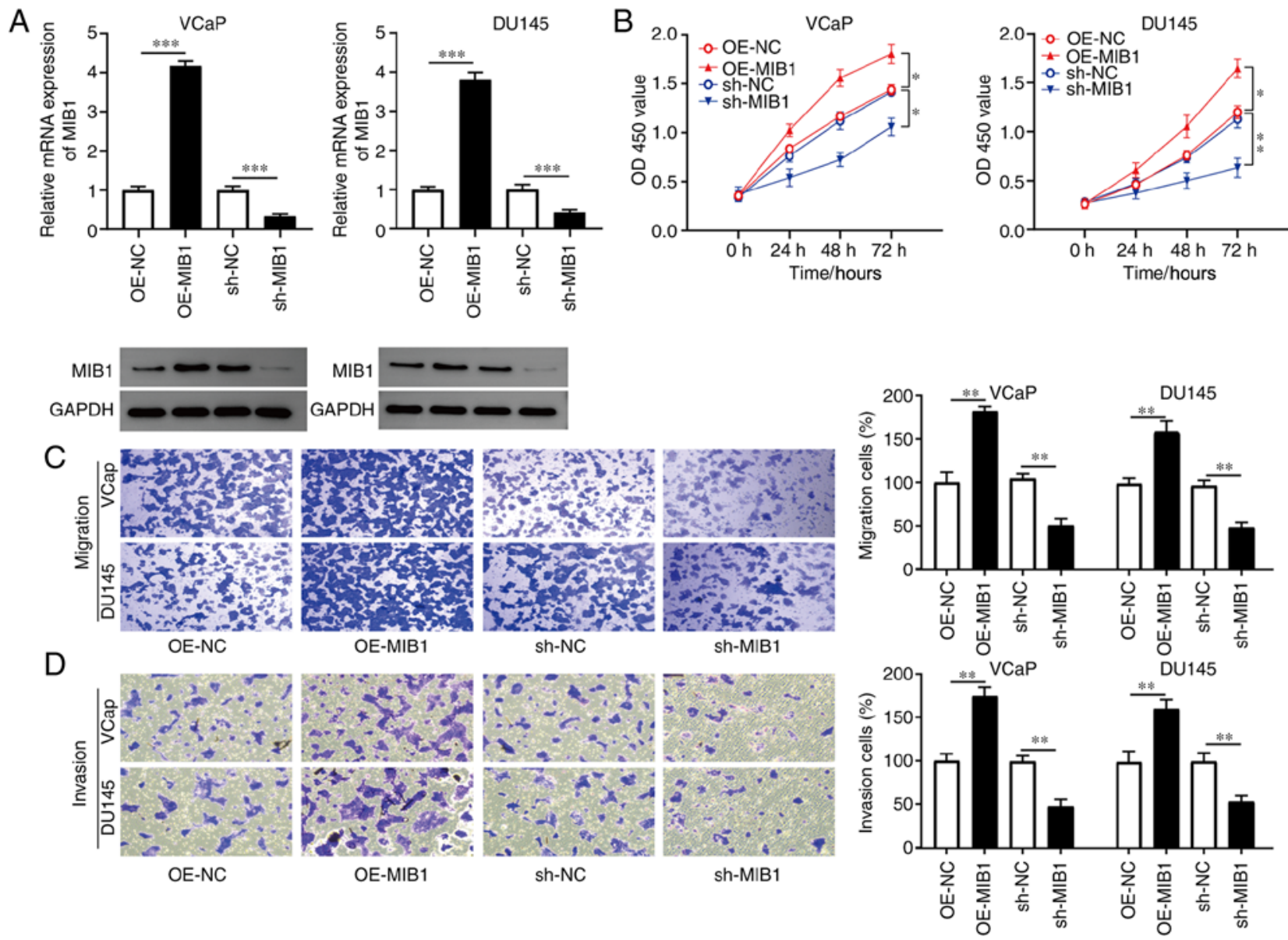

Figure 2. MIB1 promotes PCa cell proliferation, migration and invasion. To elucidate the function of MIB1 in PCa, the VCaP and DU145 cells were transfected with OE-MIB1, sh-MIB1, OE-NC or sh-NC. (A) mRNA and protein expression level of MIB1 in the transfected VCaP and DU145 cells was measured using reverse transcription-quantitative PCR and western blot analysis, respectively. (B) Cell Counting Kit- 8 assay was used to assess the proliferation of the transfected VCaP and DU145 cell lines. Transwell assays were used to evaluate the (C) migration and (D) invasion of the transfected VCaP and DU145 cell lines. "P<0.05, ${ }^{* *} \mathrm{P}<0.01$ and ${ }^{* * *} \mathrm{P}<0.001$. MIB1, mind bomb 1; PCa, prostate cancer; OE, overexpression; sh, short hairpin RNA; NC, negative control; OD, optical density.

to identify the miRNAs that could potentially target MIB1. The putative binding sites between MIB1 and miR-195-5p are presented in Fig. 3A. To confirm whether there was a direct interaction, luciferase reporter vectors for MIB1-WT and MIB1-MUT were constructed and subsequently co-transfected into the VCaP and DU145 cell lines alongside miR-195-5p mimics or miR-NC. The results indicated that transfection with miR-195-5p mimics significantly decreased the luciferase activity of the MIB1-WT reporter, but had no effect on the luciferase activity of the MIB1-MUT reporter, suggesting that there was a targeted regulatory relationship between MIB1 and miR-195-5p in both cells lines (Fig. 3B and C). Taken together, these results demonstrated that miR-195-5p could directly bind with MIB1. 
A Position 1350-1357 of MIB1 3' UTR

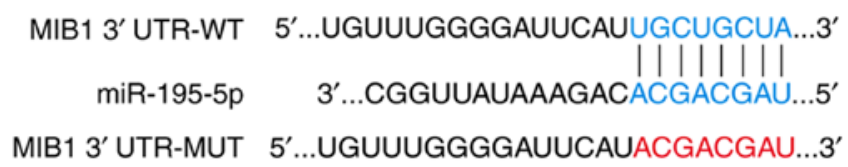

$\mathrm{B}$

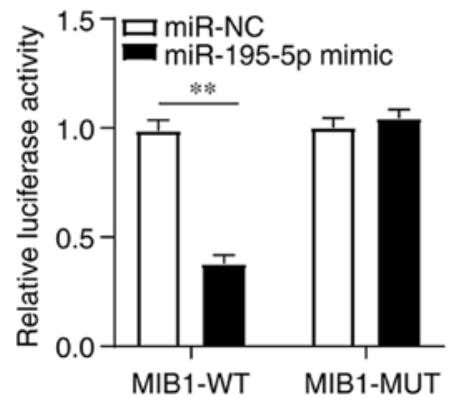

C

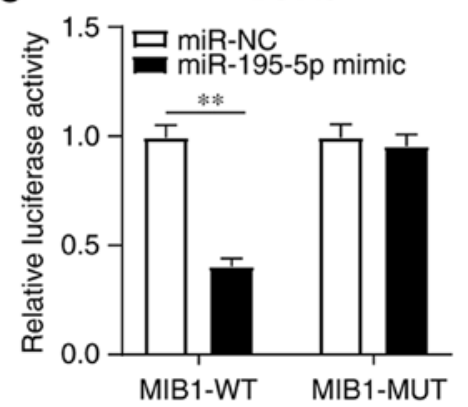

Figure 3. miR-195-5p directly interacts with MIB1. (A) Putative binding sites between miR-195-5p and MIB1, and the mutant sequences of MIB1. Luciferase activity was detected in (B) VCaP and (C) DU145 cells co-transfected with MIB1-WT or MIB1-MUT, and miR-195-5p mimics or miR-NC. ${ }^{* *} \mathrm{P}<0.01$. miR, microRNA; MIB1, mind bomb 1; WT, wild-type; MUT, mutant; NC, negative control; UTR, untranslated region.
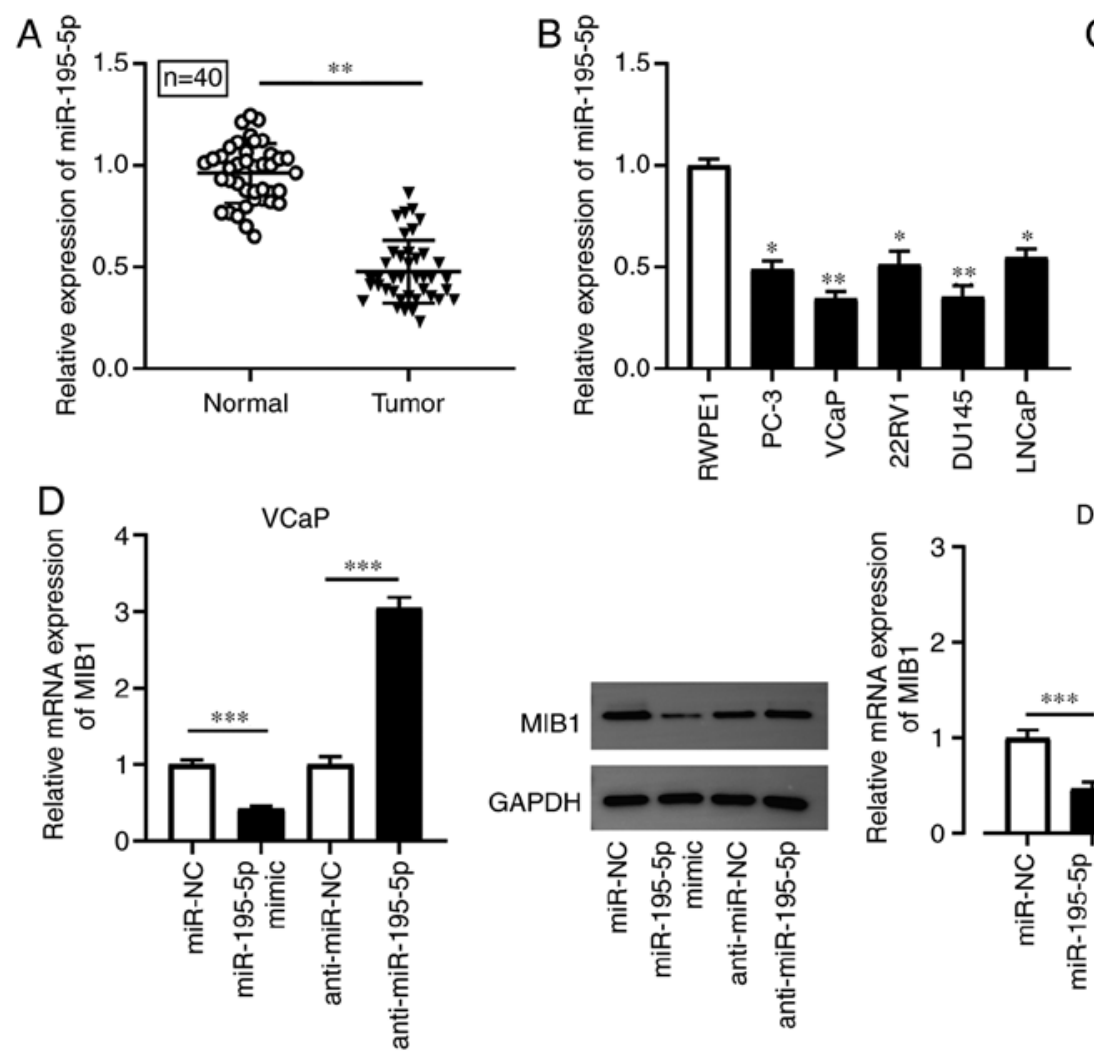

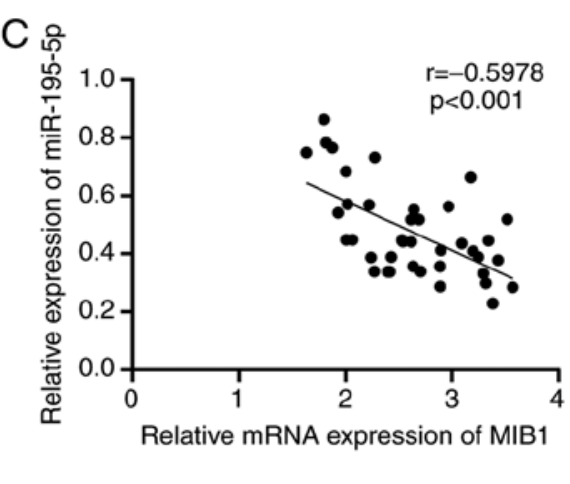

DU145

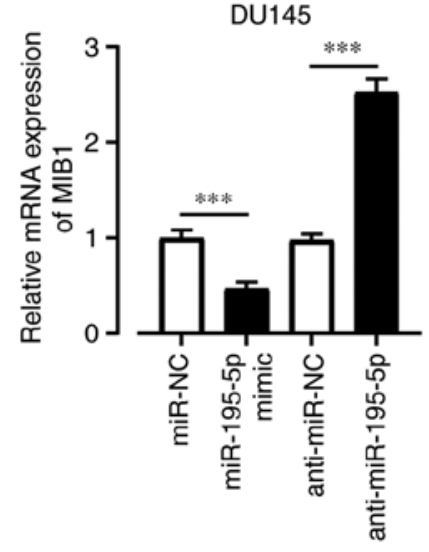

Figure 4. miR-195-5p expression level is elevated in PCa tissues and cell lines, and MIB1 is directly regulated by miR-195-5p. The mRNA expression level of miR-195-5p was examined using RT-qPCR in (A) PCa tissues and adjacent normal tissues ( $\mathrm{n}=40$ ), as well as in (B) PCa cell lines (PC-3, VCaP, 22Rv1, DU145 and LNCaP) and a normal human prostate epithelial cell line (RWPE1). (C) Pearson's analysis was used to assess the correlation between MIB1 and miR-195-5p expression in PCa. (D) MIB1 expression was measured using RT-qPCR and western blot anlaysis in VCaP and DU145 cells transfected with miR-195-5p mimics, anti-miR-195-5p, miR-NC or anti-miR-NC. ${ }^{*} \mathrm{P}<0.05,{ }^{* * *} \mathrm{P}<0.01$ and ${ }^{* * * *} \mathrm{P}<0.001$. miR, microRNA; PCa, prostate cancer; MIB1, mind bomb 1; RT-qPCR, reverse transcription-quantitative PCR; NC, negative control.

miR-195-5p inhibits the expression level of MIBI in the PCa cell lines. The mRNA expression level of miR-195-5p in the PCa tissues and cell lines (PC-3, VCaP, 22Rv1, DU145 and $\mathrm{LNCaP}$ ) was detected using RT-qPCR. The results demonstrated that miR-195-5p mRNA expression level was significantly decreased in PCa tissues $(\mathrm{n}=40)$ and cell lines (PC-3, VCaP, 22Rv1, DU145 and LNCaP) compared with that in adjacent normal tissues and the normal human prostate epithelial cell line, RWPE1, respectively (Fig. 4A and B). The correlation between miR-195-5p and MIB1 expression was 

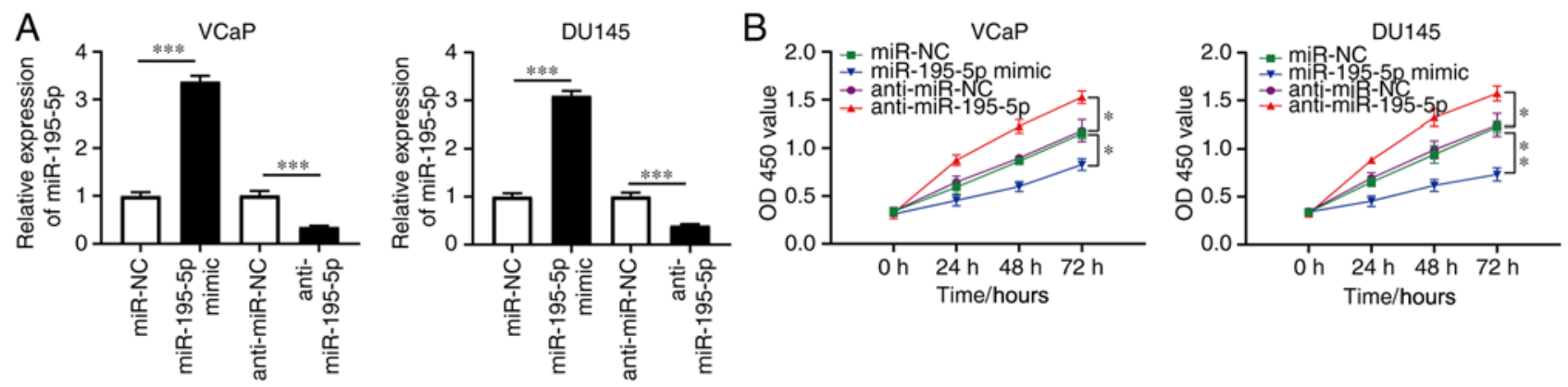

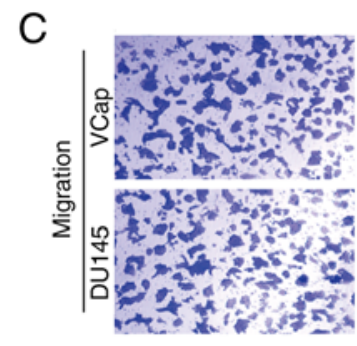

miR-NC

D

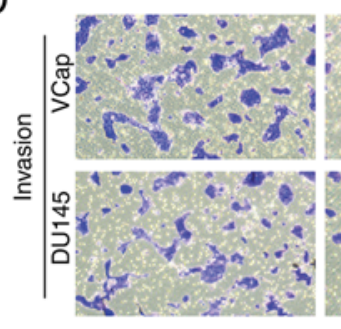

miR-NC

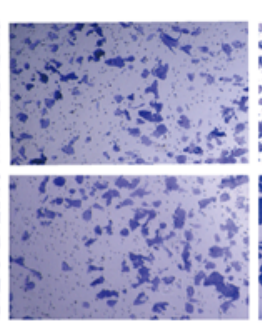

miR-195-5p

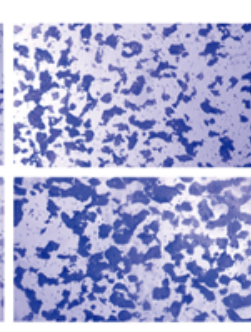

anti-miR-NC

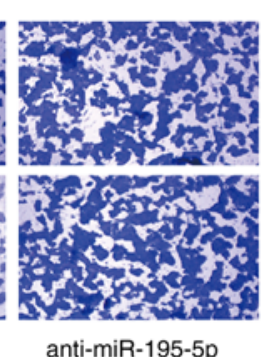

anti-miR-195-5p

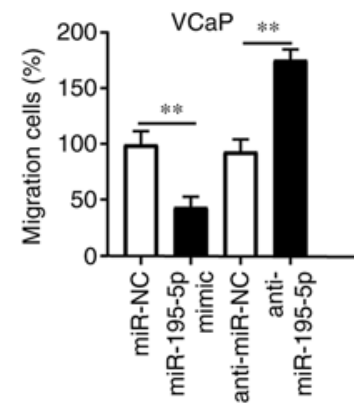

DU145

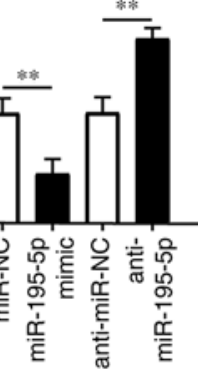

Figure 5. miR-195-5p knockdown promotes the proliferation, migration and invasion of PCa cells. Functional analysis was performed using the VCaP and DU145 cell lines transfected with miR-195-5p mimics, anti-miR-195-5p, miR-NC or anti-miR-NC. (A) miR-195-5p expression in transfected cells was measured using reverse transcription-quantitative PCR. (B) Cell Counting Kit- 8 assay was used to evaluate the proliferation of transfected VCaP and DU145 cells. Transwell assays were used to assess the (C) migration and (D) invasion of transfected $\mathrm{VCaP}$ and $\mathrm{DU} 145$ cells. ${ }^{*} \mathrm{P}<0.05$, ${ }^{* *} \mathrm{P}<0.01$ and ${ }^{* * *} \mathrm{P}<0.001$. miR, microRNA; NC, negative control; OD, optical density.

subsequently assessed using Pearson's correlation analysis. The results indicated that there was a moderate negative correlation between miR-195-5p and MIB1 mRNA expression levels (r, -0.5978; $\mathrm{P}<0.001$; Fig. 4C). The VCaP and DU145 cell lines were then transfected with miR-195-5p mimics and its inhibitor (miR-195-5p and anti-miR-195-5p) or their corresponding negative controls (miR-NC and anti-miR-NC). RT-qPCR and western blot analyses revealed that MIB1 mRNA and protein expression level, respectively, was markedly reduced in the VCaP and DU145 cell lines transfected with miR-195-5p mimics, and was notably increased following knockdown of miR-195-5p expression (Fig. 4D). These data suggested that MIB1 may serve a potential role in PCa, and that miR-195-5p suppressed the expression level of MIB1 in a targeted manner.

miR-195-5p overexpression inhibits PCa cell proliferation, migration and invasion. To investigate the biological function of miR-195-5p in PCa cells, the VCaP and DU145 cell lines were transfected with miR-195-5p mimics, anti-miR-195-5p, miR-NC or anti-miR-NC. The results of RT-qPCR demonstrated that the mRNA expression level of miR-195-5p was markedly increased in the VCaP and DU145 cells following miR-195-5p mimics transfection, and that the mRNA expression level of miR-195-5p was significantly decreased following transfection with anti-miR-195-5p (Fig. 5A). The results of the CCK-8 assay indicated that miR-195-5p mimics significantly inhibited cell proliferation, and that miR-195-5p knockdown markedly promoted the viability of the VCaP and DU145 cell lines (Fig. 5B). In addition, the results of the Transwell and Matrigel assays revealed that $\mathrm{VCaP}$ and DU145 cell migration and invasion following miR-195-5p mimics transfection was markedly reduced compared with that in the cells transfected with NC. Furthermore, miR-195-5p knockdown promoted cell migration and invasion compared with that in cells transfected with the NC (Fig. 5C and D). These data indicated that miR-195-5p regulated the proliferation, migration and invasion of PCa cells in vitro.

miR-195-5p regulates cell proliferation and invasion by promoting MIBl expression. To confirm that miR-195-5p serves a role in the regulation of MIB1, miR-195-5p and MIB1 were overexpressed and knocked down at the same time. RT-qPCR and western blot analyses revealed that MIB1 overexpression reversed the inhibition of endogenous MIB1 expression in the VCaP and DU145 cell lines transfected with miR-195-5p 

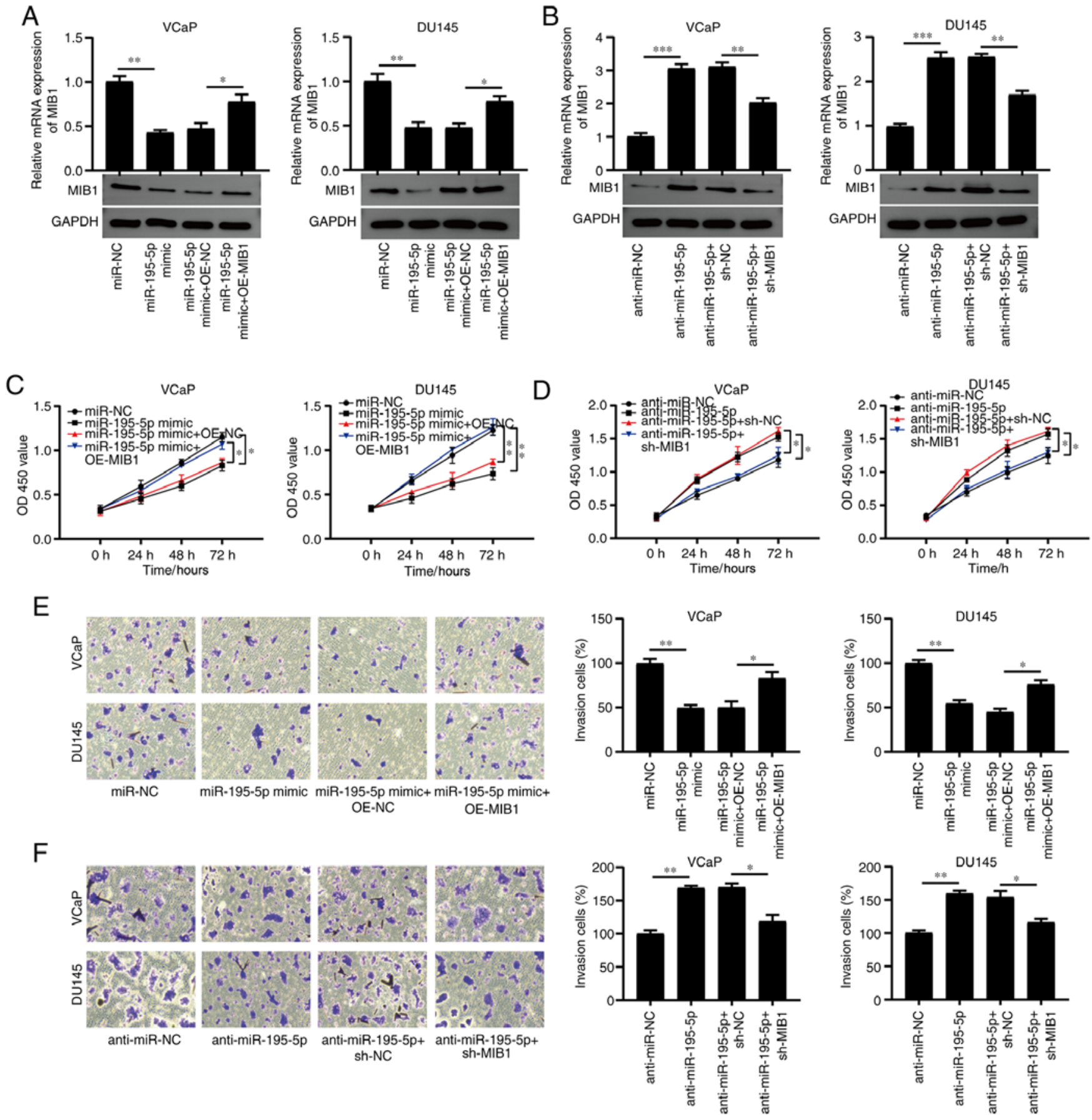

Figure 6. miR-195-5p promotes the proliferation and invasion of PCa cells via MIB1. To elucidate the function of the miR-195-5p/MIB1 axis in PCa, the VCaP and DU145 cell lines were transfected with miR-195-5p mimics, miR-NC, anti-miR-195-5p, anti-miR-NC, miR-195-5p mimics + OE-NC, miR-195-5p mimics + OE-MIB1, anti-miR-195-5p + sh-MIB1 or anti-miR-195-5p + sh-NC. The mRNA and protein expression levels of MIB1 was determined in the VCaP and DU145 cell lines using reverse transcription-quantitative PCR and western blot analysis, respectively following transfection with (A) miR-195-5p mimics and OE-MIB1, and (B) anti-miR-195-5p and sh-MIB1. Cell Counting Kit-8 assay was used to assess the proliferation of VCaP and DU145 cells transfected with (C) miR-195-5p mimics, miR-NC, miR-195-5p mimics + OE-NC, miR-195-5p mimic + OE-MIB1, and (D) anti-miR-195-5p, anti-miR-NC, anti-miR-195-5p + sh-MIB1 or anti-miR-195-5p + sh-NC. Transwell assay was used to detect the invasion of VCaP and DU145 cells transfected with (E) miR-195-5p mimics, miR-NC, miR-195-5p mimics + OE-NC, miR-195-5p mimics + OE-MIB1 and (F) anti-miR-195-5p, anti-miR-NC, anti-miR-195-5p + sh-MIB1 or anti-miR-195-5p + sh-NC. ${ }^{*} \mathrm{P}<0.05,{ }^{* *} \mathrm{P}<0.01$ and ${ }^{* * * *} \mathrm{P}<0.001$. microRNA; PCa, prostate cancer; MIB1, mind bomb 1; NC, negative control; OE, overexpression; sh, short hairpin RNA; OD, optical density.

mimics (Fig. 6A). In addition, MIB1 knockdown (sh-MIB1) reversed the promotion of MIB1 expression in the $\mathrm{VCaP}$ and DU145 cells transfected with anti-miR-195-5p (Fig. 6B). According to the results of the CCK-8 assay, miR-195-5p mimic transfection suppressed the proliferation of the $\mathrm{VCaP}$ and DU145 cell lines, and co-transfection of miR-195-5p mimics and OE-MIB1 reversed the effect of miR-195-5p mimics on cell proliferation (Fig. 6C). Anti-miR-195-5p transfection promoted the proliferation of the $\mathrm{VCaP}$ and DU145 cell lines, and the co-transfection of anti-miR-195-5p and sh-MIB1 abolished the effect of miR-195-5p knockdown on cell proliferation (Fig. 6D). The overexpression of MIB1 rescued the miR-195-5p overexpression-induced anti-invasive effects of the VCaP and DU145 cell lines (Fig. 6E) and co-transfection 
of anti-miR-195-5p and sh-MIB1 reversed the effect of miR-195-5p knockdown on cell invasion (Fig. 6F). The results demonstrated that miR-195-5p regulated cell proliferation and invasion by promoting MIB1 expression in PCa.

\section{Discussion}

PCa is considered to be a common malignant tumor, that poses a major threat to the health of men worldwide $(1,2)$. Therefore, designing novel treatment strategies is important for the effective therapy of patients with PCa. The current study aimed to identify novel biomarkers that could improve current treatment strategies. The results revealed the essential role of MIB1 in PCa cell proliferation, migration and invasion, and identified that miR-195-5p was a regulator of MIB1 expression. RT-qPCR indicated that miR-195-5p mRNA expression level was decreased in PCa tissues and cell lines, while MIB1 expression was elevated. By targeting MIB1, it was demonstrated that miR-195-5p inhibited the proliferation and invasion of PCa cells. Collectively, the findings of the current study indicated that MIB1 may be crucial for PCa progression and that miR-195-5p may inhibit the proliferation of PCa.

MIB1 is an E3 ubiquitin ligase. Previous studies have indicated that a number of molecules, such as catenin $\delta-1$ (CTNND1) (10), receptor-like tyrosine kinase (11), death-associated protein kinase (12), $\delta(14)$, Werner syndrome protein (28), Jagged (29) and polo-like kinase 4 (30), were ubiquitinated by MIB1. For example, MIB1 may directly regulate cell migration by ubiquitinating CTNND1 to modulate GTPase activity (10). The present study verified that the MIB1 mRNA and protein expression level was significantly increased in PCa tissues and cell lines (PC-3, VCaP, 22Rv1, DU145 and LNCaP). Furthermore, MIB1 overexpression markedly promoted $\mathrm{VCaP}$ and DU145 cell proliferation, migration and invasion. Bioinformatics analysis and the dual-luciferase reporter assay demonstrated that MIB1 was a direct target gene of miR-195-5p. However, further studies on the role of MIB1 and miR-195-5p in PCa are required.

Over the past few years, supporting lines of evidence have indicated that miRNAs serve crucial roles in prostate tumorigenesis using a range of mechanisms (31-36), and this has attracted the interest of numerous researchers who aim to determine their underlying mechanisms of action. miR-195-5p belongs to the miR-15 family, and has been demonstrated to exhibit a low expression level in various types of cancer, serving a key negative regulatory role (17-21). In PCa, miR-195-5p suppressed the migration and invasion of the DU145 and PC3 cell lines by targeting Fos-related antigen 1 (22). Furthermore, miR-195 inhibited the proliferation of the PC-3, LNCaP and DU145 cell lines by targeting ribosomal protein S6 kinase B1 (23). Cai et al (24) demonstrated that miR-195 inhibited DU145 and LNCaP cell proliferation and angiogenesis by downregulating the expression of proline rich 11. In addition, hsa_circular RNA_0062019 promoted the proliferation, migration and invasion of PCa cells via the miR-195-5p/high-mobility group AT-hook 2 axis (37). Long non-coding (lnc)RNA AFAP1 antisense RNA 1 has also been demonstrated to modulate the sensitivity of paclitaxel-resistant PCa cells (PC3 and DU145) to paclitaxel via the miR-195-5p/FKBP prolyl isomerase 1A axis (38). IncRNA LINC00473 additionally contributed to cell proliferation by regulating the miR-195-5p/SEPT2 axis (39). Consistent with these studies, the present study confirmed that miR-195-5p was significantly decreased in PCa tissues and cell lines (PC-3, VCaP, 22Rv1, DU145 and LNCaP), and revealed that miR-195-5p overexpression inhibited the proliferation, migration and invasion of $\mathrm{VCaP}$ and DU145 cells. The results indicated that miR-195-5p regulated cell proliferation and invasion by promoting MIB1 expression.

However, the present study still has some limitations, which require further investigation. First, whether the expression level of MIB1 and miR-195-5p is associated with reduced overall survival, recurrence-free survival or metastasis-free survival in patients with PCa should be analyzed using Kaplan-Meier survival analysis. Unfortunately, this was not achieved in the present study due to insufficient collection of relevant data. Second, future investigations are required to determine the more specific functions of MIB1 and miR-195-5p in PCa cells, such as their role in cell apoptosis with in vivo experimental verification.

In conclusion, the current study demonstrated that miR-195-5p overexpression markedly suppressed VCaP and DU145 cell proliferation, migration and invasion. The results are supported by previous studies (22-24) and indicate that miR-195-5p may serve an essential role in cancer development and progression. More importantly, the current study revealed that MIB1 overexpression markedly promoted the proliferation, migration and invasion of $\mathrm{VCaP}$ and DU145 cells. The results additionally suggested that miR-195-5p overexpression inhibited the proliferation and invasion of PCa cells by targeting MIB1, providing a novel promising molecular target for the treatment of $\mathrm{PCa}$.

\section{Acknowledgements}

Not applicable.

\section{Funding}

This research was funded by the National Natural Science Foundation of China (grant no. 31860242) and the Construction Projects of Medical Biomaterial Research and Development Talent Base in Guizhou Province and Zunyi City [grant nos. (2018)3, and (2019)69].

\section{Availability of data and materials}

The datasets used and/or analyzed during the current study are available from the corresponding author upon reasonable request.

\section{Authors' contributions}

$\mathrm{BC}$ and GB confirm the authenticity of all the raw data. BC designed the study, analyzed the experiments and wrote the paper. $\mathrm{GB}, \mathrm{XM}$ and LT collected the data and formed data analysis. HX designed the study, and wrote and revised the manuscript. All authors read and approved the final version of the manuscript.

\section{Ethics approval and consent to participate}

The present study was approved by the Zunyi Medical University Ethics Committee. All participants provided written informed consent. 


\section{Patient consent for publication}

Not applicable.

\section{Competing interests}

The authors declare that they have no competing interests.

\section{References}

1. Cai JR, Chen Z, Chen XL, Huang H, Lin X and Miao B: Coexpression network analysis identifies a novel nine-RNA signature to improve prognostic prediction for prostate cancer patients. Biomed Res Int 2020: 4264291, 2020.

2. Wild CP, Espina C, Bauld L, Bonanni B, Brenner H, Brown K, Dillner J, Forman D, Kampman E, Nilbert M, et al: Cancer prevention Europe. Mol Oncol 13: 528-534, 2019.

3. Yang Y, Chen R, Sun T, Zhao L, Liu F, Ren S, Wang H, Lu X, Gao X, Xu C and Sun Y: Efficacy and safety of combined androgen blockade with antiandrogen for advanced prostate cancer. Curr Oncol 26: e39-e47, 2019.

4. Corona G, Baldi E and Maggi M: Androgen regulation of prostate cancer: Where are we now? J Endocrinol Invest 34: 232-243, 2011

5. Damber JE and Aus G: Prostate cancer. Lancet 371: 1710-1721, 2008.

6. Steele CB, Li J, Huang B and Weir HK: Prostate cancer survival in the United States by race and stage (2001-2009): Findings from the CONCORD-2 study. Cancer 123 (Suppl 24): S5160-S5177, 2017.

7. Moyer VA, U.S. Preventive services task force: Screening for prostate cancer: U.S. Preventive services task force recommendation statement. Ann Intern Med 157: 120-134, 2012.

8. Loeb S, Bjurlin MA, Nicholson J, Tammela TL, Penson DF Carter HB, Carroll P and Etzioni R: Overdiagnosis and overtreatment of prostate cancer. Eur Urol 65: 1046-1055, 2014.

9. Welch HG and Albertsen PC: Reconsidering prostate cancer mortality-the future of PSA screening. N Engl J Med 382: $1557-1563,2020$

10. Mizoguchi T, Ikeda S, Watanabe S, Sugawara M and Itoh $M$ : Mib1 contributes to persistent directional cell migration by regulating the Ctnnd1-Rac1 pathway. Proc Natl Acad Sci USA 114: E9280-E9289, 2017.

11. Berndt JD, Aoyagi A, Yang P, Anastas JN, Tang L and Moon RT: Mindbomb 1, an E3 ubiquitin ligase, forms a complex with RYK to activate Wnt//3-catenin signaling. J Cell Biol 194: 737-750, 2011

12. Jin Y, Blue EK, Dixon S, Shao Z and Gallagher PJ: A death-associated protein kinase (DAPK)-interacting protein, DIP-1, is an E3 ubiquitin ligase that promotes tumor necrosis factor-induced apoptosis and regulates the cellular levels of DAPK. J Biol Chem 277: 46980-46986, 2002.

13. Zhang L and Gallagher PJ: Mind bomb 1 regulation of cFLIP interactions. Am J Physiol Cell Physiol 297: C1275-C1283, 2009.

14. Itoh M, Kim CH, Palardy G, Oda T, Jiang YJ, Maust D, Yeo SY, Lorick K, Wright GJ, Ariza-McNaughton L, et al: Mind bomb is a ubiquitin ligase that is essential for efficient activation of Notch signaling by delta. Dev Cell 4: 67-82, 2003.

15. Ray J, Hoey C, Huang X, Jeon J, Taeb S, Downes MR, Boutros PC and Liu SK: MicroRNA-198 suppresses prostate tumorigenesis by targeting MIB1. Oncol Rep 42: 1047-1056, 2019.

16. Hu YM, Lou XL, Liu BZ, Sun L, Wan S, Wu L, Zhao X, Zhou Q, Sun MM, Tao K, et al: TGF- $\beta 1$-regulated miR-3691-3p targets E2F3 and PRDM1 to inhibit prostate cancer progression. Asian J Androl 23: 188-196, 2021.

17. Zheng J, Xu TT, Chen F and Zhang Y: miRNA-195-5p functions as a tumor suppressor and a predictive of poor prognosis in non-small cell lung cancer by directly targeting CIAPIN1. Pathol Oncol Res 25: 1181-1190, 2019.

18. Luo JH, Pan JS, Jin Y, Li MY and Chen M: miR-195-5p inhibits proliferation and induces apoptosis of non-small cell lung cancer cells by targeting CEP55. Onco Targets Ther 12: 11465-11474, 2019.

19. Du W, Liu T, Zhang Y, Zeng Y, Zhu J, Tang H, Liu Z and Huang JA: miR-195-5p is a potential factor responsible for CPNE1 differential expression between subtypes of non-small cell lung cancer. J Cancer 11: 2610-2620, 2020

20. Liu XM, Zhou Y, Ning YE, Gu H, Tong YX and Wang N: miR-195-5p inhibits malignant progression of cervical cancer by targeting YAP1. Onco Targets Ther 13: 931-944, 2020.
21. Li M, Ren CX, Zhang JM, Xin XY, Hua T, Wang HB and Wang HB: The effects of miR-195-5p/MMP14 on proliferation and invasion of cervical carcinoma cells through TNF signaling pathway based on bioinformatics analysis of microarray profiling. Cell Physiol Biochem 50: 1398-1413, 2018.

22. Wu J, Ji A, Wang X, Zhu Y, Yu Y, Lin Y, Liu Y, Li S, Liang Z, $\mathrm{Xu}$ X, et al: MicroRNA-195-5p, a new regulator of Fra-1, suppresses the migration and invasion of prostate cancer cells. J Transl Med 13: 289, 2015

23. Cai C, Chen QB, Han ZD, Zhang YQ, He HC, Chen JH, Chen YR, Yang SB, Wu YD, Zeng YR, et al: miR-195 inhibits tumor progression by targeting RPS6KB1 in human prostate cancer. Clin Cancer Res 21: 4922-4934, 2015.

24. Cai C, He H, Duan X, Wu W, Mai Z, Zhang T, Fan J, Deng T, Zhong W, Liu Y, et al: miR-195 inhibits cell proliferation and angiogenesis in human prostate cancer by downregulating PRR11 expression. Oncol Rep 39: 1658-1670, 2018.

25. Alexandratou E, Yova D, Gorpas D, Maragos P, Agrogiannis G and Kavantzas N: Texture analysis of tissues in Gleason grading of prostate cancer. Int Soc Opt Photon 6859: 685904, 2008.

26. Ozkan TA, Eruyar AT, Cebeci OO, Memik O, Ozcan L and Kuskonmaz I: Interobserver variability in Gleason histological grading of prostate cancer. Scand J Urol 50: 420-424, 2016.

27. Livak KJ and Schmittgen TD: Analysis of relative gene expression data using real-time quantitative PCR and the 2(-Delta Delta C(T)) method. Methods 25: 402-408, 2001.

28. Li M, Liu B, Yi J, Yang Y, Wang J, Zhu WG and Luo J: MIB1-mediated degradation of WRN promotes cellular senescence in response to camptothecin treatment. FASEB J 34: 11488-11497, 2020.

29. Koo BK, Lim HS, Song R, Yoon MJ, Yoon KJ, Moon JS, Kim YW, Kwon MC, Yoo KW, Kong MP, et al: Mind bomb 1 is essential for generating functional Notch ligands to activate Notch. Development 132: 3459-3470, 2005.

30. Cajánek L, Glatter T and Nigg EA: The E3 ubiquitin ligase Mib1 regulates Plk4 and centriole biogenesis. J Cell Sci 128: 1674-1682, 2015.

31. Zhang X, Zhou J, Xue D, Li Z, Liu Y and Dong L: miR-515-5p acts as a tumor suppressor via targeting TRIP13 in prostate cancer. Int J Biol Macromol 129: 227-232, 2019.

32. Zhan B, Huang L, Chen Y, Ye W, Li J, Chen J, Yang S and Jiang W: miR-196a-mediated downregulation of p27 protein promotes prostate cancer proliferation and relates to biochemical recurrence after radical prostatectomy. Prostate 80: 1024-1037, 2020.

33. Yang Y, Jia B, Zhao X, Wang Y and Ye W: miR-93-5p may be an important oncogene in prostate cancer by bioinformatics analysis. J Cell Biochem 120: 10463-10483, 2019.

34. Nam RK, Benatar T, Wallis CJD, Kobylecky E, Amemiya Y, Sherman C and Seth A: MicroRNA-139 is a predictor of prostate cancer recurrence and inhibits growth and migration of prostate cancer cells through cell cycle arrest and targeting IGF1R and AXL. Prostate 79: 1422-1438, 2019.

35. Du Y, Liu XH, Zhu HC, Wang L, Ning JZ and Xiao CC: miR-543 promotes proliferation and epithelial-mesenchymal transition in prostate cancer via targeting RKIP. Cell Physiol Biochem 41: 1135-1146, 2017

36. Xu F, Li Q, Wang ZY and Cao XM: Sinomenine inhibits proliferation, migration, invasion and promotes apoptosis of prostate cancer cells by regulation of miR-23a. Biomed Pharmacother 112: 108592,2019

37. Wang P, Zhang L, Yin S, Xu Y, Tai S, Zhang LI and Liang C: hsa_circ_0062019 promotes the proliferation, migration, and invasion of prostate cancer cells via the miR-195-5p/HMGA2 axis. Acta Biochim Biophys Sin (Shanghai) 12: 815-822, 2021.

38. Leng W, Liu Q, Zhang S, Sun D and Guo Y: lncRNA AFAP1-AS1 modulates the sensitivity of paclitaxel-resistant prostate cancer cells to paclitaxel via miR-195-5p/FKBP1A axis. Cancer Biol Ther 21: 1072-1080, 2020 .

39. Xing ZS, Li SL, Liu ZX, Zhang C, Meng MJ and Bai ZM: The long non-coding RNA LINC00473 contributes to cell proliferation via JAK-STAT3 signaling pathway by regulating miR-195-5p/SEPT2 axis in prostate cancer. Biosci Rep 40: BSR20191850, 2020.

This work is licensed under a Creative Commons Attribution-NonCommercial-NoDerivatives 4.0 International (CC BY-NC-ND 4.0) License. 